\title{
A Brassinosteroids-BES1 Regulatory Module Manipulate Thermomemory in Arabidopsis thaliana
}

\author{
xiuhong yao ${ }^{1}$, Juan $\mathrm{Chen}^{1}$, Wen $\mathrm{Yu}^{1}$, Zuxu Zhou ${ }^{1}$, Fabin Yang ${ }^{1}$, Yanling $\mathrm{Li}^{1}$, Dawei \\ Zhang $^{1}$, and HONG-HUI LIN ${ }^{1}$ \\ ${ }^{1}$ Sichuan University State Key Laboratory of Hydraulics and Mountain River Engineering
}

March 4, 2022

\begin{abstract}
Heat stress (HS) caused by ambient high temperature pose a threat to plants. In the natural and agricultural environment, plants often encounter repeated and changeable HS. Moderate HS primes plants to establishment of a molecular 'thermomemory' that enables plants to withstand a later-and possibly more extreme-HS attack. Recent years, brassinosteroids (BRs) have been implicated in HS response whereas little is known about whether BRs signal transduction modulates thermomemory. Here, we uncover the positive role of BRs signaling in thermomemory of Arabidopsis thaliana. Heat priming induces de novo synthesis and nuclear accumulation of BRI1-EMS-SUPPRESSOR (BES1), the key regulator of BRs signaling. BRs promote the accumulation of dephosphorylated BES1 during memory phase, blocking BRs synthesis impairs dephosphorylation. During HS memory, BES1 is required to maintain sustained induction of HS memory genes and directly targets APX2 and HSFA3 for activation. In summary, our results reveal a BES1-required, BRs-enhanced transcriptional control module of thermomemory in Arabidopsis thaliana.
\end{abstract}

\section{INTRODUCTION}

Due to climate change, the global temperature will continue rising in the future, which will cause great challenges to crop production (Lesk, Rowhani, \& Ramankutty, 2016). Ambient temperature of plants increases rapidly by 10 will pose a threat to plants and cause heat stress (HS). In order to survive, plants form a complex and efficient regulatory network to resist and adapt to HS, understanding these protective responses has important agronomic value for maximizing agricultural production and maintaining yield (Huang, Zhao, Burger, Wang, \& Chory, 2021). Under HS, heat shock transcription factors (HSFs) are induced rapidly to active the expression of heat shock proteins (HSPs). HSPs mainly function as molecular chaperones to protect against thermal denaturation of substrates and facilitate refolding of denatured substrates, by regulating heat shock proteins, plants can tolerate certain heat levels (McLoughlin, Kim, Marshall, Vierstra, \& Vierling, 2019).

In the natural environment, plants often suffer from repeated and changeable heat shock, these sub-lethal HS induce plants to produce thermal memory (Balmer, Pastor, Gamir, Flors, \& Mauch-Mani, 2015). HS priming endows plants with a stronger thermal response to a second stress exposure. Hs memory involves an initial priming phase and a memory period that lasts for several days (Ohama, Sato, Shinozaki, \& YamaguchiShinozaki, 2017). The initial phase of the HS memory involves interacting transcriptional regulatory networks, HSFs play fundamental role among these transcription factors. In addition, specific modifications in the chromatin enable these memory genes to be induced rapidly to cope with next stress in the memory phase (Lamke, Brzezinka, Altmann, \& Baurle, 2015). During the HS memory state, several HSPs with chaperone activity maintain high protein levels for several days after priming (Wu et al., 2013).

As efficient and accurate signal molecules, phytohormones actively participate in HS response. Exogenous 
application of abscisic acid (ABA) increases hydrogen peroxide $\left(\mathrm{H}_{2} \mathrm{O}_{2}\right)$ accumulation by inducing the expression of respiratory burst oxidase Homologs (RBOHs), and thus enhances antioxidant capacity (Suzuki et al., 2011). In addition, ABA also induces the expression levels of HSPs to maintain protein in functional conformations (X. Wang, Zhuang, Shi, \& Huang, 2017). In flower tissue of Arabidopsis , cytokinin treatment increases resistance in response to harmful high temperature, protecting developing flowers exposed to HS (K. Wang, Zhang, \& Ervin, 2012). Salicylic acid (SA) reduces heat stress-induced membrane damage, enhancing the activities of antioxidant enzymes (Shah Jahan et al., 2019). In addition, SA treatment maintains the level of HSP21 protein in chloroplast, facilitates the recovery of photosynthesis after stress (L. J. Wang et al., 2010). High temperatures lead to accumulation of jasmonate (JA), loss of function in JA signaling or biosynthesis impairs the thermotolerance of Arabidopsis seedlings, indicating that JA is required for effective heat tolerance (Clarke et al., 2009). JA treatment did not induce the expression of HSPs, and a defect in JA signaling has no impact on HSPs transcription level (Manvi \& Ashverya, 2016). In contrast to JA, ethylene signal transduction pathway promotes the expression of HSFs and HSPs via ETHYLENE RESPONSE FACTOR 95/97 (ERF95/97), the direct target of ETHYLENE INSENSITIVE 3 (EIN3) (Huang et al., 2021). Despite overwhelming evidences supporting the vital role phytohormones act in HS response, limited knowledge of the molecular mechanism restricts the application in crops, it is urgent to identify pivotal factors in response to heat shock in phytohormones signaling.

Brassinosteroids (BRs) are a class of plant steroid hormones that play a central role in the control of plant growth and development (Nolan, Nemanja, Liu, Eugenia, \& Yin, 2019). BRs are perceived by the transmembrane receptor kinase BRASSINOSTEROID INSENSITIVE 1 (BRI1), and the binding of BRs activates BRI1, thus triggering a phosphorylation cascade and finally inactivating BR-INSENSITIVE 2 (BIN2), a GSK3-like kinase that negatively modulates BRs signal by phosphorylating BRI1-EMS-SUPPRESSOR 1 (BES1) and BRASSINAZOLE RESISTANT 1 (BZR1), the key regulators of BR-regulated gene expression (Guo, Li, Aluru, Aluru, \& Yin, 2013). Transcriptome analysis showed that BES1/BZR1 up- or down-regulate thousands of BR-responsive genes (Sun et al., 2010; Yu. et al., 2011). In the spring barley (Hordeum vulgare $L$.), improved functioning of PSII was observed under HS upon 24-epibrassinolide (EBR) application (Janeczko, Okleková, Pociecha, Kocielniak, \& Mirek, 2011). In rice (Oryza sativa), EBR or 7,8-dihydro-820-hydroxyecdysone (DHECD) application improves photosynthetic efficiency and raises the rice yield under HS (Diaz et al., 2021). In tomato, epi-brassinolide (EBL) induces thermotolerance and peroxidase (POD) activity (Mazorra, Holton, Bishop, \& Nunez, 2011). HS promotes the dephosphorylation of BES1 through repressing the activity of type $2 \mathrm{C}$ phosphatases (PP2Cs), activated BES1 directly binds to heat shock elements (HSEs) thus contribute to HS signaling (Albertos et al., 2022). These studies demonstrate that BRs signal play a positive role in HS response. Nevertheless, little is known about whether BRs signal transduction modulates thermal memory process, so far there are no reports involved in BR-mediated thermomemory.

In this study, we report that BRs act positively in HS memory and that BES1 is required for BRs-enhanced thermomemory. Heat priming induces the nucleus accumulation of BES1 and BES1 protein sustains for high levels several days after priming. BRs treatment further activates BES1 during the memory phase. The ability to maintain high levels of BES1 protein in the nucleus after priming is required for sustained activation of HS memory-associated genes and BES1 activates memory genes in a direct or indirect manner. Our results thus demonstrate that BES1 lays the foundation of thermomemory, while BRs further pull up the upper limit of it.

\section{RESULTS}

\subsection{BRs play a positive role in thermomemory}

To elucidate the role of BRs in thermomemory in Arabidopsis, we performed a thermotolerance assay under primed and unprimed conditions towards wild-type Col-0. Heat priming and triggering protocol was outlined in Fig. 1A. Seedlings grown on standard half-strength Murashige-Skoog (1/2MS) medium with or without $10 \mathrm{nM} \mathrm{BL}$ (brassinolide, a kind of BRs with high physiological activity) were vibrant before HS. Upon HS triggering and recovery, seedlings grown on the medium containing BL exhibited significantly enhanced thermotolerance, accompanied with comparably elevated survival rate and decreased electrolyte 
leakage. Thermomemory induced by heat priming effectively improved the thermotolerance of seedlings and the application of BL further enhanced the vitality of thermomemory (Fig. 1). These observations suggest that BRs positively modulate thermomemory process.

\subsection{BES1 is specifically required for thermomemory}

Given that BRs act positively in thermomemory, we then investigated whether the defect in BRs synthesis weakened memory response. Previous studies reported that BR-deficient and -overproduction seedlings represented similar thermal tolerance (Albertos et al., 2022; Mazorra et al., 2011), to further investigate whether endogenous BRs levels affect thermomemory, we subject 5-day-old det2 and DET2-OX seedlings to our priming and triggering stimulus protocol. Surprisingly, both det2 and DET2-OX represented enhanced thermal tolerance (Supplementary Fig. 1), indicating that thermomemory is also independent of BR homeostasis, this promotes us to explore downstream of BRs signaling. We next access the role BES1 acts in thermomemery since the effect of BRs is largely mediated by the BES1/BZR1 subfamily transcription factors. Considering the functional redundancy between BES1 and BZR1, we chose BES1-RNAi (a mutant BES1/BZR1 mRNA almost undetectable caused by RNA interference) to carry out thermomemory assay (Yin et al., 2005). As shown in Fig. 1, BES1 deficiency caused seedlings hypersensitive to heat, as comparably declined survival rate and increased electrolyte leakage was detected in BES1-RNAi . In contrast to the elevated thermotolerance by priming stimulation in Col-0, primed BES1-RNAi seedlings represent similar thermotolerance to the unprimed, indicating that BES1/BZR1 is required for thermomemory. Application of $10 \mathrm{nM}$ BL increased the survival rate of Col-0 seedlings, however, it had no obvious effect on BES1-RNAiunder both primed and unprimed conditions. These results suggest that BES1 is indispensable for BRs-enhanced thermomemory.

\subsection{BES1 accumulates during the memory phase}

To explore the expression pattern of BES1 in the memory phase, we carried out a time course towards seedling collected at different time points indicated in Fig. 2A. The transcriptional level of BES1 remained high until $48 \mathrm{~h}$ into the thermomemory phase, and still more than 6 fold compared with the control in 48 h (Fig. 2B). Consistent with this, GUS analysis also demonstrates that heat priming inducible expression of BES1 (Fig. 2C). Next, we assessed whether the increased mRNA level in the memory phase leads to the accumulation of BES1. We investigated the abundance of BES1 during thermomemory assay and observed more than 2-fold accumulation in the memory phase compared with control (prior priming), and BES1 protein remains abundant until $48 \mathrm{~h}$ into the thermomemory phase (Fig. 2D). To test whether the sustained high level of BES1 protein at later memory time points are due to the rise of transcriptional level and requires de novo protein synthesis, we applied cycloheximide (CHX) to inhibit protein translation. Col-0 seedlings were treated with CHX after priming and BES1 protein level was determined by immune blotting at 12 $\mathrm{h}, 24 \mathrm{~h}$ and $48 \mathrm{~h}$ into the memory phase. Compared with mock treatment (DMSO), BES1 protein level declined in the memory phase (Fig. 2E), supporting our suspect that increases in BES1 mRNA contribute to the accumulation of BES1 protein during the memory phase. In addition, we also concerned about the effect caused by protein degradation, since the increased BES1 may also come from the contribution of weakening degradation. Compared with mock treatment, treatment with MG132 resulted in an increase of BES1 within the memory phase (Fig. 2F), indicating that the degradation of BES1 weakened during that time. Furthermore, we carried out a combined treatment with Col-0 seedling using both CHX and MG132. Not surprisingly, BES1 abundance in these seedlings was slightly higher than that of CHX treatment but obvious lower than MG132 treatment (Fig. 2G). These results demonstrate that de novo synthesis and weakened degradation mutually contribute to the accumulation of BES1 during the memory phase.

\subsection{Heat priming and BRs promote the accumulation of dephosphorylated BES1 during mem- ory phase}

HS activates BES1 via dephosphorylation (Albertos et al., 2022), so we are curious about whether the state can be sustained in the memory phase. We executed a transient expression experiment on $N$. benthamiana leaves to explore the subcellular distribution of BES1 after heat priming. As shown in Fig. 3A, BES1 
was diffused in the nucleus and cytoplasm prior priming, after exposure to 44 for 90 min, more BES1GFP was shuttled to the nucleus, and the accumulation of BES1 in the nucleus could be maintained for another 2-3 d. BRs signal transduction activates BES1/BZR1 through releasing the inhibition of BIN2 thus promotes dephosphorylation of BES1, so we suppose BRs mediate thermomemory through promoting the accumulation of activated BES1 during the memory phase. We detected the abundance of de-phosphorylated BES1 in seedlings grown on solid plate medium with or without BL application during the memory phase. As shown in Fig. 3B, BL application strengthens the accumulation of dephosphorylated BES1, whereas BRZ (brassinazole, a specific inhibitor of BR synthesis) treatment impairs dephosphorylation. These results indicate that heat priming promotes the accumulation of dephosphorylated BES1 in the nucleus, BRs signal transduction further enhances this process.

\subsection{The abundance of dephosphorylated BES1 is responsible for BR-enhanced thermomemory}

To elucidate the critical function of BES1 accumulated during thermal memory phase for BR signal, we detected BES1 protein by immunoblot analysis in det2 and BES1-RNAi seedlings at $48 \mathrm{~h}$ of memory phase. As shown in Supplementary Fig. 2, the abundance of dephosphorylated BES1 was higher in det2, whereas it was almost undetectable in BES1-RNAi . This finding provides further evidence for BES1 involved in the regulation of thermomemory, increased BES1 level in det2 could explain the enhanced thermotolerance indet2 mutant (Supplementary Fig. 1) and a lack of BES1 could be responsible for the thermomemory function-loss in BES1-RNAi (Fig. 2).

We next subjected 5-day-old bes1-D mutant and Col-0 seedlings to our priming and triggering stimulus protocol, since the amino acid mutation in bes1-D results in an increase in the total amount of BES1 protein. Not surprisingly, dephosphorylated BES1 in bes1-Dis comparably more abundant than that of Col-0 during the whole memory phase. Consistent with this observation, bes1- $D$ seedlings revealed better thermotolerance than Col-0 in both unprimed and primed conditions, as the survival rate was comparably higher than Col-0 (Fig. 4C), accompanied with lower electrolyte leakage in bes1-D (Fig. 4D), underscoring the contribution of bio activated BES1 to elevating the thermolerance and enhance thermomemory.

\subsection{BES1 is required for sustained activation of HS-associated memory genes}

To analyze whether BES1 mediates thermomemery through affecting the expression of HS-associated memory genes, we examined the transcript level of memory genes in thermomemory assay at the indicating time point. The HS memory-associated genes APX2 (Stief et al., 2014a),HSFA3 (Friedrich et al., 2021), HSA32 (Wu et al., 2013),HSP21 (Sedaghatmehr, Mueller-Roeber, \& Balazadeh, 2016) were analyzed (include both type I and type II HS-associated memory gene) and a non-memory but HS-inducible gene HSP101 (McLoughlin et al., 2019) was set as contrast. These genes did not show dramatic differences among Col-0, bes1-D and BES1-RNAi prior priming (Fig. 5A), indicating that BES1 has a weak regulatory effect on these genes under normal growth conditions. The expression of APX2 , HSFA3 and HSP21 was induced several hundred-fold (Fig. 5B, D and E) and HSA32 for several dozen fold (Fig. 5C) early in the memory phase and sustained relative high transcript level $3 \mathrm{~d}$ after priming in wild-type and bes1- $D$. These genes were less induced in BES1-RNAi mutant early in the memory phase and later decreased faster than Col-0 and bes1-D. With the deepening of memory phase, the expression of these memory genes started to decrease between 12 and $24 \mathrm{~h}$ and declined dramatically $48 \mathrm{~h}$ after priming in BES1-RNA $i$ when it was still relatively high in Col-0 and bes1-D . For the non-memory gene HSP101, no significant difference was found among Col-0, bes1-D and BES1-RNAi during the memory phase (Fig. $5 \mathrm{~F}$ ). These results indicate that BES1 is required to a great extent for sustained activation of HS memory-associated genes.

\subsection{BES1 directly targets $A P X 2$ and $H S F A 3$ in the memory phase}

Since BES1 is critical for the sustained activation of HS-associated memory genes, we further examine whether BES1 directly target these genes for activation. Yeast one-hybrid (Y1H) assays indicate that BES1 directly activates the expression of APX2 and HSFA3 (Fig. 6A). We next conducted an electrophoretic mobility shift assay (EMSA) towards the fragment of APX2 and HSFA3 promoter containing an E-box, the potential BES1 protein binding site. As expected, the EMSA assay showed that MBP-BES1 proteins could 
bind to APX2 and HSFA3 probes, but MBP did not (Fig. 6B, C). We further confirmed that BES1 directly binds to the promoter of $A P X 2$ and $H S F A 3$ in vivo using a chromatin immunoprecipitation (ChIP)-qPCR assay. Obvious enrichment was found in the promoter of $A P X 2$ and $H S F A 3$ (Fig. 6D). These results suggest that APX2 and HSFA3 are transcriptional targets of BES1 proteins during the thermomemory process.

\section{Discussion}

Global temperatures are increasing due to global warming, which greatly increases the probability of extreme high temperatures happening, these changes substantially disturb normal crop growth and yield around the world, which seriously affects human food security (Hasanuzzaman, Nahar, Alam, Roychowdhury, \& Fujita, 2013). Facing the changeable and repetitive stress environment, plants have evolved 'thermopriming' mechanisms which enable them to respond more effectively to a second stress exposure. The molecular 'memory' can last for several days and this stress-free period is so-called the thermomemory phase. HS memory involves the regulation of transcription factors, epigenetic modification of chromatin, post-transcriptional modifications of proteins, metabolic control and coordinated regulation at multiple organ level (Balazadeh, 2021). Priming of stress-induced gene expression is critical for stress priming (Brzezinka, Altmann, \& Baurle, 2019). In this study, we reported that BES1 is crucial for long-term maintenance of thermal memory genes (Fig. 5). MiRNA156 sustains expression of thermomemory genes such as HSA32 and APX2 in a post-transcriptional regulation manner (Stief et al., 2014b). In addition, BRUSHY1 (BRU1)/TONSOKU/MGOUN3 maintains the sustained induction of memory genes through mediating the epigenetic inheritance of chromatin states (Brzezinka et al., 2019). Our work provides evidence for BRs signal enhanced control module for these memory genes continuous induction, thus uncover a new sight of phytohormone molecule-affected transcription cascade control of thermomemory.

Although the importance of Brassinosteroids in elevating thermotolerance in Arabidopsis and crops has already been reported, the details of the molecular mechanisms BRs signal plays in thermomemory remain largely unexplored. We still do not fully understand how BRs control a large number of heat response genes, when this regulation occurs, which master regulator orderly controls the responses to environmental heat cues (Nawaz et al., 2017). A recent study indicates that a defect in BES1 showed serious sensitive characteristics to heat stress compared to wild type. Heat shock rapidly activates BES1 and ABA promotes the dephosphorylation of BES1 through repressing the activity of PP2C-type phosphatases in HS process (Albertos et al., 2022). HSP90 forms a complex with BES1 and assists the compartmentalized cycle between active and inactive BES1 (Samakovli et al., 2020; Shigeta et al., 2015). In this study, we further discovered that BES1 accumulation in the nucleus in response to heat priming and sustained for another 2-3 days into the memory phase (Fig. 2). The molecular mechanism was further supported by genetic evidence of the heat-resistant phenotype of bes1-D and heat-intolerant BES1-RNAi mutant. Thus, we additionally found the new performance of elaborate regulator BES1.

We observed that BES1 was still degraded slowly under the combined treatment of CHX and MG132, especially later in the memory phase when the memory response is gradually weakening (Fig. $2 \mathrm{~F}$ ), indicating other protein degradation pathways also target BES1 for degradation, such as the lysosome and autophagy pathway. Since autophagy plays a crucial role in nutrient cycling and tolerance to various biotic and abiotic stress (Nishad \& Nandi, 2021), it is interesting to explore the interaction between autophagy and BRs signals in response to heat stress, especially in controlling the balance between BES1 degradation and stability. The phosphorylated and dephosphorylated BES1 were degraded mutually in the majority of cases. Thus an increased de novo protein synthesis is required for BES1 accumulation in the nucleus during heat stress. BZR1 accumulates in the nucleus at high temperature, inducing the expression of growth-promoting genes such as PHYTOCHROME-INTERACTING FACTOR 4 (PIF4) thus facilitating plant growth under high temperature (Ibanez et al., 2018). Similarly, our research found that heat priming induces the transcription of BES1 and finally contributes to BES1 accumulation in the nucleus during thermomemory phase (Fig. 2B, C). Most research focuses on the phosphorylation and degradation of BES1, given the importance of BES1 transcript for protein accumulation, it is crucial to elucidate the upstream signal that establishment and controls the transcriptional activation of BES1 in heat stress and thermomemory response. 
BRs act to mitigate heat stress via compensation and priming of gene expression. BR treatment enhances reactive oxygen species scavenging to alleviate the impacts of high temperatures by inducing production of SOD and POD (Yin et al., 2018), but specific molecular mechanisms that activate the expression of these enzymes are largely unknown. A research performed exogenous EBR treatment with Arabidopsis BRs mutants and found loss-of-function in either BRs biosynthesis or signaling exhibit increased ROS accumulation (Setsungnern et al., 2020). Nevertheless, the concrete molecular mechanism by which BRs signal transduction activates ROS scavenging remains to be revealed. According to the ChIP-chip results, Ascorbate Peroxidase 1(APX1) and APX3 are two putative targets of BES1 (Yu. et al., 2011). In this study, we identified APX2 directly regulated by BES1 during thermomemory phase, providing a possible molecular mechanism for BR-regulated ROS scavenging when plants suffer from heat attack.

Studies in Arabidopsis and tomato demonstrate that downstream of BRs signaling, but not BRs level, make sense in heat stress response (Mazorra et al., 2011; Setsungnern et al., 2020). Similarly, a latest study reveals that BES1 is rapidly dephosphorylated and activated by heat stress in a manner independent of BRs signaling (Albertos et al., 2022). It seems like a paradox that BRs activate BES1/BZR1 but endogenous BRs levels are indispensable for thermal tolerance. Interestingly, cytosolic BES1/BZR1 can be recruited to the nucleus following activation of BRs, whereas environmental factors affect the protein abundance of BES1/BZR1, thus BRs-BES1 regulatory module is critical for plants to integrate environmental factors and endogenous signals to maximize survival (R. Wang et al., 2021; Yang et al., 2017). BRs treatment significantly improved heat tolerance in tomato (Nie et al., 2013), maize (Yadava, Kaushal, Gautam, Parmar, \& Singh, 2016) and barley (Janeczko et al., 2011). Our study provides a BR independent but enhanced thermomemory response. The increasing frequencies of heatwaves poses a great threat to crop yields and food supplies, fortunately, exogenous supply of stress-signaling phytohormones like BRs benefit to mitigate the negative effects of high temperature in plants. Thus, the knowledge BRs function in thermomemory is helpful for breeding or editing genomes of agricultural crops.

In summary, our results provide insight into BR-enhanced thermomemory in plants. Although endogenous BRs level is indispensable for thermotolerance, exogenous application of BRs can certainly enhance basal thermotolerance and thermomemory, in a BES1 dependent manner. BES1 responds to heat priming and accumulates in the nucleus to activate the expression of HS-associated memory genes. This finding is beneficial for manipulating the BR pathway in crops and other plants so that plants can accommodate the heat stress created by frequent harmful high temperature fluctuations in these environmental parameters.

\section{MATERIALS AND METHODS}

\subsection{Plant materials}

DET2-OX , bes1-D and det2 used in this study were previously reported (Zhang et al., 2021) and BES1RNAi (Li et al., 2020). 35S::GFP, 35S::BES1-GFP and proBES1::GUS were introduced into Col-0 by Agrobacterium tumefaciens -mediated floral transformation. All the plants were in the Col-0 ecotype.

\subsection{Thermotolerance assay}

Seeds were sown on $1 / 2$ MS plates with $0.70 \%$ agar and stratified at 4 for $3 \mathrm{~d}$. Then the plates were moved to the long day growth chamber (16-h light and 8-h dark, $120 \mu \mathrm{mol} \cdot \mathrm{m}^{-2} \cdot \mathrm{s}^{-1}$, LED fluorescent tube) at 22. Priming treatment was performed as described previously with subtle modifications(Brzezinka et al., 2019). Briefly, Seedlings grown in a chamber for $5 \mathrm{~d}$ were subjected to priming protocol: $37 \mathrm{deg} \mathrm{C}$ for $60 \mathrm{~min}$, followed by $120 \mathrm{~min}$ at $22 \mathrm{degC}$, then $45 \mathrm{~min}$ at $44 \mathrm{degC}$ followed $2 \mathrm{~d}$ at 22 , then seedlings were subjected to $44 \mathrm{degC}$ for $90 \mathrm{~min}$ in a constant-temperature incubator for HS triggering. For the unprimed group, triggering was applied to seedlings grown at $22 \mathrm{degC}$ for $7 \mathrm{~d}$. After triggering, seedlings were transferred to normal growth conditions until analysis.

\subsection{Electrolyte leakage assay}

To measure electrolyte leakage caused by high temperature, seedlings were harvested before or after heat triggering and rinsed twice with deionized water, placed into 10-mL tubes containing $8 \mathrm{~mL}$ of deionized 
water. Samples were placed at room temperature for $24 \mathrm{~h}$ and measured the conductivity using an Electroconductivity meter (DDSJ-308A, Lei-ci, Shanghai, China), recorded as R1. Samples were then boiled at $100 \mathrm{deg} C$ for $15 \mathrm{~min}$ and shaken at room temperature for another $1 \mathrm{~h}$ before the electrical conductivity was measured again, recorded as R2. The electrolyte leakage was calculated as: R1/R2. Three biological replicates were performed for per simple.

\subsection{RNA isolation and RT-qPCR analysis}

Total RNA was isolated from seedlings using an RNeasy Mini Kit (Qiagen) according to the manufacturer's instructions, and the extracted RNA was treated with DNase to remove potential genomic DNA contamination. First-strand cDNA was synthesized using M-MLV Reverse Transcriptase (Invitrogen) with approximately $2 \mu \mathrm{g}$ RNA. RT-qPCR reactions were conducted on a $20 \mu \mathrm{l}$ total reaction volume containing $10 \mu \mathrm{l}$ of SYBR Green Real-Time PCR Master Mix (Transgene, Beijing, China) and $2 \mu$ cDNA. The CFX96 Real-Time PCR Detection system (Bio-Rad, Hercules, CA, USA) was used for qRT-PCR analysis. The relative expression levels of genes were normalized to the reference gene ACTIN2 (AT3G18780). For each sample, three biological replicates were performed and each biological replicate contained three technical replicates.

\subsection{GUS staining}

The promoter of bes1 was cloned into PBI121 vector and introduced into Col-0 by Agrobacterium tumefaciens-mediated floral transformation to generate proBES1::GUS transgenic plant. Five-dayoldproBES1::GUS was grown in long day growth chamber (16-h light and 8-h dark, $120 \mu \mathrm{mol} . \mathrm{m}^{-2} \cdot \mathrm{s}^{-1}$, LED fluorescent tube) at 22 , then seedlings were subjected to priming assays and sampled at different time points. Seedlings were stained at 37 in the dark for $2.5 \mathrm{~h}$ using GUS Staining Solution (Sangon Biotech, Cat no. A610085-0025, Shanghai). After staining, the seedlings were washed with $75 \%$ ethanol and the image was captured using a fluorescence microscope (Leica, Wetzlar, Germany).

\subsection{Protein extraction and western blot analysis}

For protein extraction, about $50 \mathrm{mg}$ of tissue samples were placed in a $2 \mathrm{ml}$ plastic centrifugal pipe and grinded into fine powder in liquid nitrogen, then added $150 \mu \mathrm{L}$ protein loading buffer ( $5 \%$ sodium dodecyl sulphate, $0.25 \%$ bromophenol blue, $2.5 \%$-mercaptoethanol, $25 \%$ glycerol) to the powder and boiled at $100^{\circ} \mathrm{C}$ for $10 \mathrm{~min}$ followed by centrifugation at $13000 \mathrm{~g}$ for $10 \mathrm{~min}$ at $4^{\circ} \mathrm{C}$. Clear supernatants was used as total protein extract. The extracted protein solution was electrophoresed on $10 \%$ sodium dodecyl sulphate (SDS)-gel and transferred to the nitrocellulose membrane to incubate with antibody. For BES1 protein accumulation analysis, Proteins from different samples were detected by anti-BES1 (Yin's lab) at a dilution of $1: 5000$.

\subsection{Subcellular localization}

The whole-genome sequence of BES1 was amplified and cloned into the pCAMBIA1302-GFP vector to generate proBES1::BES1-GFP. The vector was transformed into Agrobacterium tumefaciens strain (GV3101) and transient transformation into $N$. benthamiana leaves. After $48 \mathrm{~h}$, the leaves were subjected to heat priming treatment, and the GFP fluorescence was detected at different time points using a fluorescence microscope (Leica, Wetzlar, Germany).

\subsection{Electrophoretic mobility shifts assay (EMSA)}

EMSA were performed as previously described (Lei et al., 2020). Constructs for MBP-BES1 (Li et al., 2020) were transformed intoEscherichia coli strain BL21 (DE3) and recombinant proteins were induced by IPTG. MBP-BES1 was purified using Amylose resin (Cat no. E8021; New England Biolabs, Ipswich, MA, USA). Purified MBP-BES1 was incubated with the probe listed in supplemental table, the mixture was reaction in binding buffer (25 mM HEPES-KOH, pH 8.0, $50 \mathrm{mM} \mathrm{KCl,} 1 \mathrm{mM}$ dithiothreitol and $10 \%$ glycerol) for 30 min and shift in polyacrylamide gel. The shifted probe and protein were tested with EMSA Kit (Thermo Fisher). 


\subsection{Yeast one-hybrid (Y1H) assays}

The full-length CDS of BES1 was cloned into the PGAD-T7 vector and the promoter of APX2, HSFA3, HSA32, HSP21 were cloned into pHIS2 vector. The constructs and empty vector controls were transformed into yeast strain Y187 and grown on SD/-Leu-Trp-His dropout plates for interaction analysis.

\subsection{Chromatin immunoprecipitation (ChIP)-qPCR}

ChIP was performed as previously described (Zhang et al., 2021). In brief, Arabidopsis seedlings harvest at $12 \mathrm{~h}$ after priming were collected and crosslinked with formaldehyde and used for chromatin isolation. After ultrasonic crushing, the complex of chromatins and GFP-BES1 or GFP were immunoprecipitated by anti-GFP antibody and harvested by protein A beads. Then the purified DNA was quantified by RT-qPCR using specific primer listed in supplemental table.

\section{ACCESSION NUMBER}

BES1 (AT1G193500) DET2 (AT2G38050) HSA32 (AT4G21320)HSP21 (AT4G27670) HSP22 (AT4G10250) HSP101 (AT1G74310)APX2 (AT3G09640) HSFA3 (AT5G03720)

\section{ACTIN2 (AT3G18780)}

\section{ACKNOWLEDGMENTS}

We thank Yanhai Yin (Iowa State University) for providing anti-BES1. This work was supported by grants from the National Natural Science Foundation of China (grant no. 31970263 to H.H.L; 32070213 to D.W.Z); the Institutional Research Fund of Sichuan University (2020SCUNL212); and the Fundamental Research Funds for the Central Universities (SCU2020D003).

\section{REFERENCES}

Albertos, P., Dundar, G., Schenk, P., Carrera, S., Cavelius, P., Sieberer, T., \& Poppenberger, B. (2022). Transcription factor BES1 interacts with HSFA1 to promote heat stress resistance of plants.Embo Journal, e108664. doi:10.15252/embj.2021108664

Balazadeh, S. (2021). A 'hot' cocktail: The multiple layers of thermomemory in plants. Curr Opinion in Plant Biology, 65 , 102147. doi:10.1016/j.pbi.2021.102147

Balmer, A., Pastor, V., Gamir, J., Flors, V., \& Mauch-Mani, B. (2015). The 'prime-ome': towards a holistic approach to priming. Trends in Plant Science, 20 (7), 443-452.

Brzezinka, K., Altmann, S., \& Baurle, I. (2019). BRUSHY1/TONSOKU/MGOUN3 is required for heat stress memory. Plant Cell Environment, 42 (3), 771-781. doi:10.1111/pce.13365

Clarke, S. M., Cristescu, S. M., Miersch, O., Harren, F. J. M., Wasternack, C., \& Mur, L. A. J. (2009). Jasmonates act with salicylic acid to confer basal thermotolerance in Arabidopsis thaliana. New Phytologist

Diaz, K., Espinoza, L., Carvajal, R., Silva-Moreno, E., Olea, A. F., \& Rubio, J. (2021). Exogenous Application of Brassinosteroid 24-Norcholane 22(S)-23-Dihydroxy Type Analogs to Enhance Water Deficit Stress Tolerance in Arabidopsis thaliana. International Journal of Molecular Sciences, 22 (3). doi:10.3390/ijms22031158

Friedrich, T., Oberkofler, V., Trindade, I., Altmann, S., Brzezinka, K., Lamke, J., . . . Baurle, I. (2021). Heteromeric HSFA2/HSFA3 complexes drive transcriptional memory after heat stress in Arabidopsis. Nat Commun, 12 (1), 3426. doi:10.1038/s41467-021-23786-6

Guo, H., Li, L., Aluru, M., Aluru, S., \& Yin, Y. (2013). Mechanisms and networks for brassinosteroid regulated gene expression. Current Opinion in Plant Biology, 16 (5), 545-553. doi:10.1016/j.pbi.2013.08.002

Hasanuzzaman, M., Nahar, K., Alam, M., Roychowdhury, R., \& Fujita, M. (2013). Physiological, Biochemical, and Molecular Mechanisms of Heat Stress Tolerance in Plants. International Journal of Molecular Sciences, 


\section{$14(5), 9643-9684$.}

Huang, J., Zhao, X., Burger, M., Wang, Y., \& Chory, J. (2021). Two interacting ethylene response factors regulate heat stress response.Plant Cell, 33 (2), 338-357. doi:10.1093/plcell/koaa026

Ibanez, C., Delker, C., Martinez, C., Burstenbinder, K., Janitza, P., Lippmann, R., . . Quint, M. (2018). Brassinosteroids Dominate Hormonal Regulation of Plant Thermomorphogenesis via BZR1. Current Biology, 28 (2), 303-310. doi:10.1016/j.cub.2017.11.077

Janeczko, A., Okleková, J., Pociecha, E., Kocielniak, J., \& Mirek, M. (2011). Physiological effects and transport of 24-epibrassinolide in heat-stressed barley. Acta Physiologiae Plantarum, 33 (4), 1249-1259.

Lamke, J., Brzezinka, K., Altmann, S., \& Baurle, I. (2015). A hit-and-run heat shock factor governs sustained histone methylation and transcriptional stress memory. The EMBO Journal, 35 (2).

Lei, W., Li, Y., Yao, X., Qiao, K., Wei, L., Liu, B., . . . Lin, H. (2020). NAP is involved in GA-mediated chlorophyll degradation and leaf senescence by interacting with DELLAs in Arabidopsis. Plant Cell Reports, 39 (1), $75-87$.

Lesk, C., Rowhani, P., \& Ramankutty, N. (2016). Influence of extreme weather disasters on global crop production. nature, 529 (7584), 84 .

Li, T., Lei, W., He, R., Tang, X., Han, J., Zou, L., . . Z Zhang, D. (2020). Brassinosteroids regulate root meristem development by mediating BIN2-UPB1 module in Arabidopsis. PLoS Genetics, 16 (7), e1008883. doi:10.1371/journal.pgen.1008883

Manvi, S., \& Ashverya, L. (2016). Jasmonates: Emerging Players in Controlling Temperature Stress Tolerance. Frontiers in Plant Science, 6, 1129.

Mazorra, L. M., Holton, N., Bishop, G. J., \& Nunez, M. (2011). Heat shock response in tomato brassinosteroid mutants indicates that thermotolerance is independent of brassinosteroid homeostasis. Plant Physiol Biochem, 49 (12), 1420-1428. doi:10.1016/j.plaphy.2011.09.005

McLoughlin, F., Kim, M., Marshall, R. S., Vierstra, R. D., \& Vierling, E. (2019). HSP101 Interacts with the Proteasome and Promotes the Clearance of Ubiquitylated Protein Aggregates. Plant Physiology, 180 (4), 1829-1847. doi:10.1104/pp.19.00263

Nawaz, F., Naeem, M., Zulfiqar, B., Akram, A., Ashraf, M. Y., Raheel, M., . . . Aurangzaib, M. (2017). Understanding brassinosteroid-regulated mechanisms to improve stress tolerance in plants: a critical review.Environmental Science and Pollution Research International, 24 (19), 15959-15975. doi:10.1007/s11356017-9163-6

Nie, W. F., Wang, M. M., Xia, X. J., Zhou, Y. H., Shi, K., Chen, Z., \& Yu, J. Q. (2013). Silencing of tomato RBOH1 and MPK2 abolishes brassinosteroid-induced $\mathrm{H}(2) \mathrm{O}(2)$ generation and stress tolerance.Plant Cell Environment, 36 (4), 789-803. doi:10.1111/pce.12014

Nishad, A., \& Nandi, A. K. (2021). Recent advances in plant thermomemory. Plant Cell Report, 40 (1), 19-27. doi:10.1007/s00299-020-02604-1

Nolan, T. M., Nemanja, V., Liu, D., Eugenia, R., \& Yin, Y. (2019). Brassinosteroids: Multidimensional Regulators of Plant Growth, Development, and Stress Responses[OPEN]. The Plant Cell (2), 2.

Ohama, N., Sato, H., Shinozaki, K., \& Yamaguchi-Shinozaki, K. (2017). Transcriptional Regulatory Network of Plant Heat Stress Response. Trends in Plant Science, 22 (1), 53-65. doi:10.1016/j.tplants.2016.08.015

Samakovli, D., Roka, L., Plitsi, P. K., Kaltsa, I., Daras, G., Milioni, D., \& Hatzopoulos, P. (2020). Active BR signalling adjusts the subcellular localisation of BES1/HSP90 complex formation. Plant Biology, 22 (1), 129-133. doi:10.1111/plb.13040 
Sedaghatmehr, M., Mueller-Roeber, B., \& Balazadeh, S. (2016). The plastid metalloprotease FtsH6 and small heat shock protein HSP21 jointly regulate thermomemory in Arabidopsis. Nat Communcations, 7 , 12439. doi:10.1038/ncomms12439

Setsungnern, A., Munoz, P., Perez-Llorca, M., Muller, M., Thiravetyan, P., \& Munne-Bosch, S. (2020). A defect in BRI1-EMS-SUPPRESSOR 1 (bes1)-mediated brassinosteroid signaling increases photoinhibition and photo-oxidative stress during heat stress in Arabidopsis. Plant Science, 296 , 110470. doi:10.1016/j.plantsci.2020.110470

Shah Jahan, M., Wang, Y., Shu, S., Zhong, M., Chen, Z., Wu, J., . . Guo, S. (2019). Exogenous salicylic acid increases the heat tolerance in Tomato (Solanum lycopersicum L) by enhancing photosynthesis efficiency and improving antioxidant defense system through scavenging of reactive oxygen species. Scientia Horticulturae, $247,421-429$.

Shigeta, T., Zaizen, Y., Sugimoto, Y., Nakamura, Y., Matsuo, T., \& Okamoto, S. (2015). Heat shock protein 90 acts in brassinosteroid signaling through interaction with BES1/BZR1 transcription factor. Journal of Plant Physiology, 178 , 69-73. doi:10.1016/j.jplph.2015.02.003

Stief, A., Altmann, S., Hoffmann, K., Pant, B. D., Scheible, W. R., \& Baurle, I. (2014a). Arabidopsis miR156 Regulates Tolerance to Recurring Environmental Stress through SPL Transcription Factors. Plant Cell, 26 (4), 1792 .

Stief, A., Altmann, S., Hoffmann, K., Pant, B. D., Scheible, W. R., \& Baurle, I. (2014b). Arabidopsis miR156 Regulates Tolerance to Recurring Environmental Stress through SPL Transcription Factors. Plant Cell, 26 (4), 1792-1807. doi:10.1105/tpc.114.123851

Sun, Y., Fan, X. Y., Cao, D. M., Tang, W., He, K., Zhu, J. Y., . . . Oh, E. (2010). Integration of Brassinosteroid Signal Transduction with the Transcription Network for Plant Growth Regulation in Arabidopsis.Developmental Cell, 19 (5), 765-777.

Suzuki, N., Miller, G., Morales, J., Shulaev, V., Torres, M. A., \& Mittler, R. (2011). Respiratory burst oxidases: the engines of ROS signaling. Current Opinion in Plant Biology, 14 , 691-699. doi:doi: 10.1016/j.pbi.2011.07.014

Thussagunpanit, J., Jutamanee, K., Sonjaroon, W., Kaveeta, L., Chai-Arree, W., Pankean, P., \& Suksamrarn, A. (2015). Effects of brassinosteroid and brassinosteroid mimic on photosynthetic efficiency and rice yield under heat stress. Photosynthetica, 53 (2), 312-320. doi:10.1007/s11099-015-0106-5

Wang, K., Zhang, X., \& Ervin, E. (2012). Antioxidative responses in roots and shoots of creeping bentgrass under high temperature: Effects of nitrogen and cytokinin. Journal of Plant Physiology, 169 (5), 492-500.

Wang, L. J., Fan, L., Loescher, W., Wei, D., Liu, G. J., Cheng, J. S., . . Li, S. H. (2010). Salicylic acid alleviates decreases in photosynthesis under heat stress and accelerates recovery in grapevine leaves. Bmc Plant Biology, 10 (1), 34.

Wang, R., Wang, R., Liu, M., Yuan, W., Zhao, Z., Liu, X., . . Tang, W. (2021). Nucleocytoplasmic trafficking and turnover mechanisms of BRASSINAZOLE RESISTANT1 in Arabidopsis thaliana. Proc Natl Acad Sci US A, 118 (33). doi:10.1073/pnas.2101838118

Wang, X., Zhuang, L., Shi, Y., \& Huang, B. (2017). Up-Regulation of HSFA2c and HSPs by ABA Contributing to Improved Heat Tolerance in Tall Fescue and Arabidopsis. International Journal of Molecular Sciences, 18 (9), 1981.

Wu, T. Y., Juan, Y. T., Hsu, Y. H., Wu, S. H., Liao, H. T., Fung, R., \& Charng, Y. Y. (2013). Interplay between Heat Shock Proteins HSP101 and HSA32 Prolongs Heat Acclimation Memory Posttranscriptionally in Arabidopsis. Plant Physiology, 161 (4), 2075-2084. 
Yadava, P., Kaushal, J., Gautam, A., Parmar, H., \& Singh, I. (2016). Physiological and Biochemical Effects of 24-Epibrassinolide on Heat-Stress Adaptation in Maize (Zea mays L.). Natural Science, 8 (4), 171-179.

Yang, M., Li, C., Cai, Z., Hu, Y., Nolan, T., Yu, F., . . Wang, X. (2017). SINAT E3 Ligases Control the Light-Mediated Stability of the Brassinosteroid-Activated Transcription Factor BES1 in Arabidopsis.Dev Cell, 41 (1), 47-58 e44. doi:10.1016/j.devcel.2017.03.014

Yin, Y., Qin, K., Song, X., Zhang, Q., Zhou, Y., Xia, X., \& Yu, J. (2018). BZR1 Transcription Factor Regulates Heat Stress Tolerance Through FERONIA Receptor-Like Kinase-Mediated Reactive Oxygen Species Signaling in Tomato. Plant Cell Physiol, 59 (11), 2239-2254. doi:10.1093/pcp/pcy146

Yin, Y., Vafeados, D., Tao, Y., Yoshida, S., Asami, T., \& Chory, J. (2005). A new class of transcription factors mediates brassinosteroid-regulated gene expression in Arabidopsis. Cell, 120 (2), 249-259. doi:10.1016/j.cell.2004.11.044

Yu., X., J., Z., M., A., Ye., H., A., F., Guo., H., . . Yin., Y. (2011). A brassinosteroid transcriptional network revealed by genome-wide identification of BESI target genes in Arabidopsis thaliana. The Plant Journal, 65 , 634-646.

Zhang, D., Tan, W., Yang, F., Han, Q., Deng, X., Guo, H., . . Lin, H. (2021). A BIN2-GLK1 Signaling Module Integrates Brassinosteroid and Light Signaling to Repress Chloroplast Development in the Dark.Dev Cell, 56 (3), 310-324 e317. doi:10.1016/j.devcel.2020.12.001
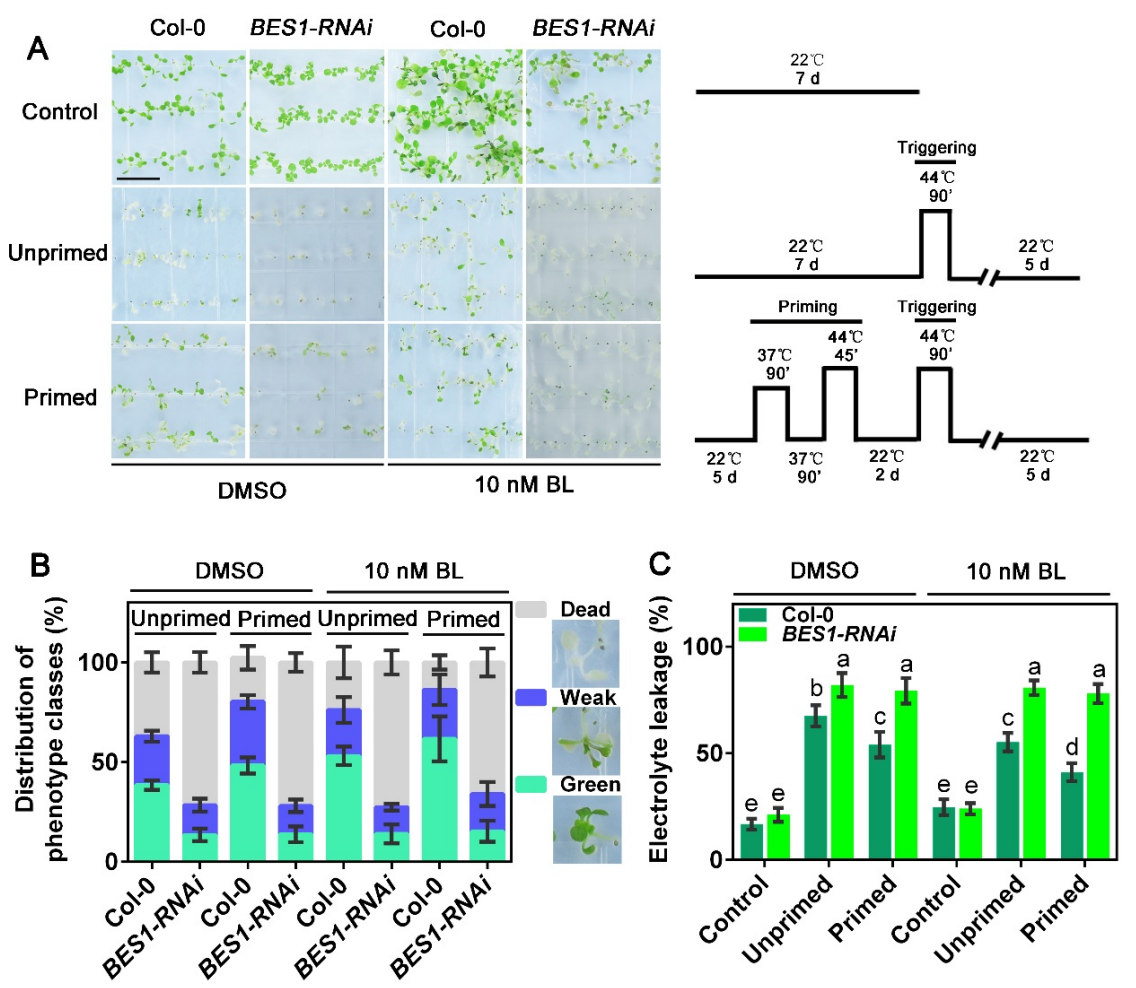

Figure 1. BES1 is required for BR-enhanced thermotolerance and memory response .

(A). Phenotype of Col-0 and BES1-RNAi under unprimed and primed conditions with or without BL application. Control: seedlings prior triggering; unprimed: seedlings were subjected to heat stress triggering and extended recovery; primed: seedlings were subjected to heat priming and stress triggering then extended 
recovery. The heat stress priming and triggering protocol was represented as schematic on the 554 right. Scale bar, $15 \mathrm{~mm}$.

(B). Percentage of seedlings grown on 1/2 MS medium with or without $10 \mathrm{nM}$ BL in different phenotype classes indicated in A. Phenotype classes were counted reference to(Sedaghatmehr et al., 2016), in short, 'green' represents the entire plant was almost green, 'weak' represents plants that were largely pale. Data shown are averages of three independent biological replicates each containing at least 100 individuals per experience group.

(C). Electrolyte leakage assay of Col-0 and BES1-RNAi grown on 1/2 MS with or without $10 \mathrm{nM}$ BL in thermotolerance assay indicated in A. Values are mean $\pm \mathrm{SEM}$ of three biological replicates. Different letters indicate statistically significant differences $(\mathrm{P}<0.05)$ compared with the control group (unprimed) exposed to the same treatment time as determined by two-way ANOVA followed by Fisher's LSD test.
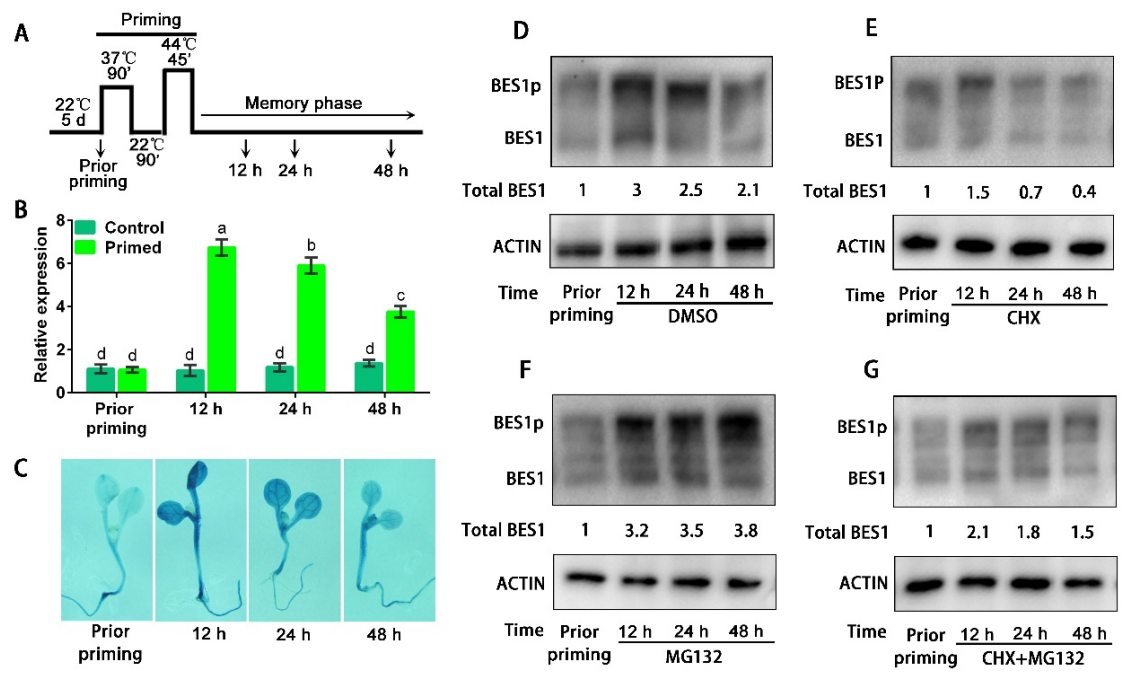

Figure 2. De novo synthesis and weakened degradation contribute to the accumulation of BES1 during the memory phase.

(A). Schematic representation of the thermomemory experimental set-up inArabidopsis .

(B). Quantitative reverse transcription-PCR revealed enhanced transcript of BES1 in the memory phase, transcripts of BES1 were normalized to ACTIN2. Values are mean \pm SEM of three biological replicates. Different letters indicate statistically significant differences $(\mathrm{P}<0.05)$ compared with the control group (unprimed) exposed to the same treatment time as determined by two-way ANOVA followed by Fisher's LSD test.

(C). BES1 promoter-GUS expression pattern in transgenic Arabidopsis plants during thermomemory assays.

(D-G). Immunoblot analysis of BES1 protein in Col-0 seedlings during the thermomemory experiment. Seedlings were treated with DMSO (C), $100 \mu \mathrm{M}$ CHX (D), $50 \mu \mathrm{M}$ MG132 (E), $100 \mu \mathrm{M}$ CHX+50 $\mu$ M MG132 (F) after priming, samples were collected at the indicated time points. Quantified relative band intensity of BES1 protein was listed below using Image J. $\beta$-ACTIN in total protein extracts was used as a loading control. 

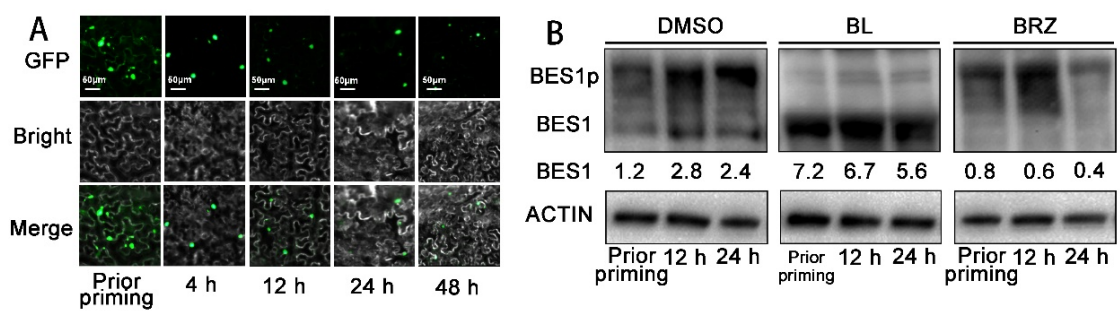

Figure 3. Heat priming and BRs promote the accumulation of dephosphorylate BES1 during thermomemory phase.

(A). BES1-GFP is accumulated to the nucleus during memory phase in leaf epidermal cells of $N$. benthamiana transformed with proBES1::BES1-GFP vector.

(B). Immuno-blot analysis of dephosphorylated BES1 abundance using $\alpha$-BES1 during thermomemory experiment in Col-0 plants grown on different medium. Quantified relative band intensity of dephosphorylated BES1 protein was listed below using Image J. $\beta$-ACTIN in total protein extracts was used as a loading control.
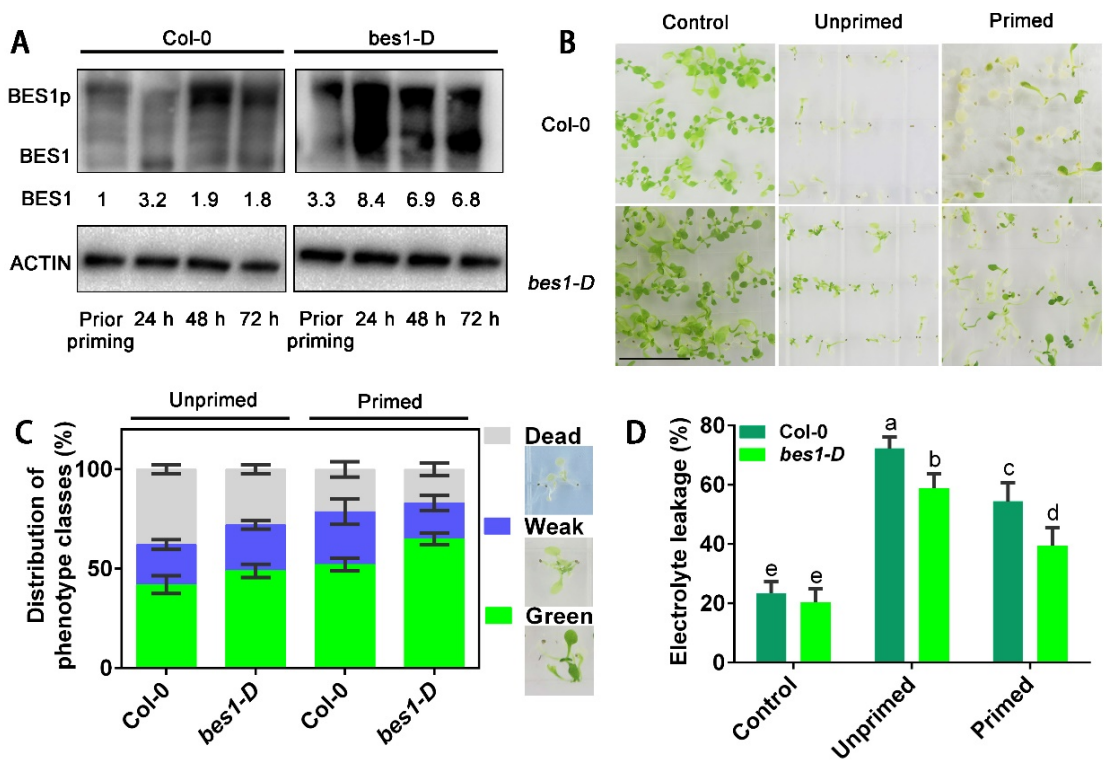

Figure 4. Increased BES1 accumulation in bes1-D enhances thermal memory.

(A). Immuno-blot analysis of BES1 abundance using $\alpha$-BES1 in col-0 andbes1-D during memory phase. Quantified relative band intensity of dephosphorylated BES1 protein was listed below using Image J. $\beta$ ACTIN in total protein extracts was used as a loading control.

(B). Phenotype of Col-0 and bes1-D under unprimed and primed conditions after recovery. Control: seedlings prior triggering; unprimed: seedlings were subjected to heat stress triggering and extended recovery; primed: seedlings were subjected to heat priming and stress triggering then extended recovery. Scale bar, $15 \mathrm{~mm}$.

(C). Percentage of seedlings in different phenotype classes indicated in A. Phenotype classes were counted reference to (Sedaghatmehr et al., 2016), in short, 'green' represents the entire plant was almost green, 'weak' 
represents plants that were largely pale. Data shown are averages of three independent biological replicates each containing at least 100 individuals per experience group.

(D). Electrolyte leakage assay of seedlings indicated in A. Values are mean \pm SEM of three biological replicates. Different letters indicate statistically significant differences $(\mathrm{P}<0.05)$ compared with the control group (unprimed) exposed to the same treatment time as determined by two-way ANOVA followed by Fisher's LSD test.
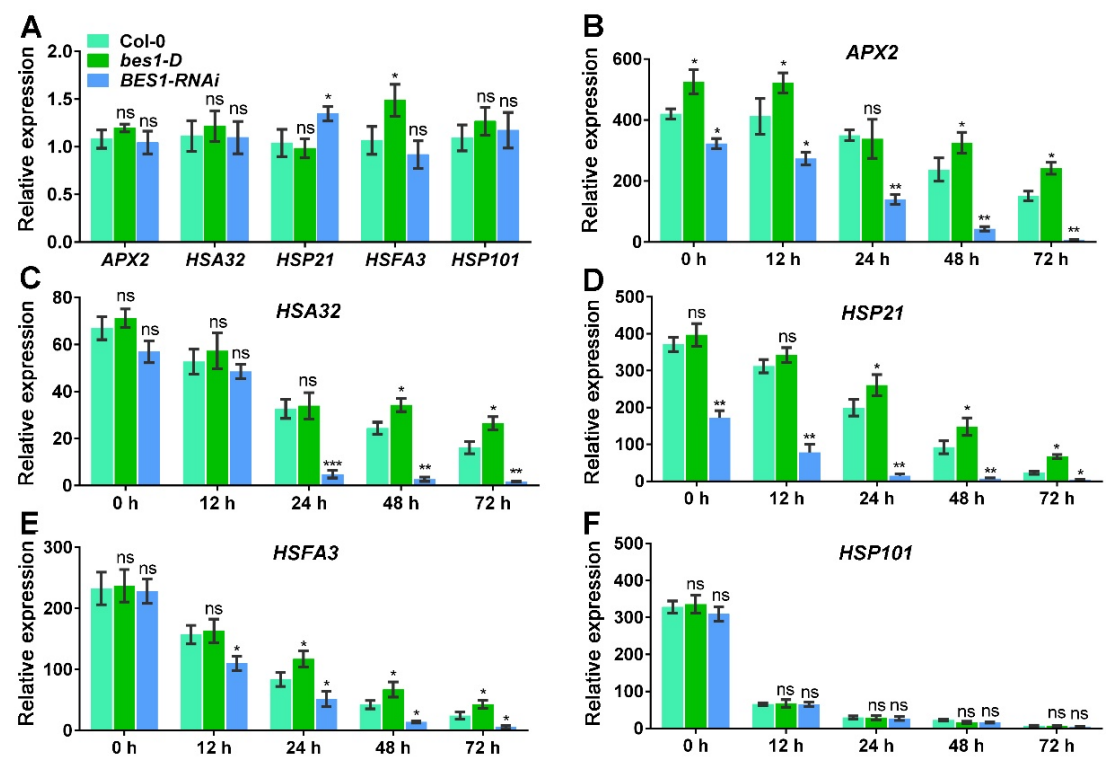

Figure 5. Sustained induction of several memory genes after priming is impaired in BES1$R N A i$ but enhanced in bes1-D.

(A). Transcript levels of memory-associated genes (APX2, HSA32, HSP21, HSFA3 ) and non-memory gene HSP101 prior priming in Col-0,bes1-D and BES1-RNAi . Values are mean \pm SEM of three biological replicates. Asterisks indicate statistically significant differences $\left({ }^{*} \mathrm{P}<0.05,{ }^{* *} \mathrm{P}<0.01,{ }^{* * *} \mathrm{P}<0.001\right)$ compared with the wild-type determined student's t test, ns indicates no significance.

(B-F). Transcript levels of HS memory-associated genes (APX2, HSA32, HSP21, HSFA3 ) and non-memory gene HSP101 after priming in Col-0, bes1-D and BES1-RNAi. Transcript levels determined by qRT-PCR were normalized to the reference gene ACTIN2 and the respective non-HS control was harvested at the same time point. Values are mean +- SEM of three biological replicates. Asterisks indicate statistically significant differences $\left({ }^{*} \mathrm{P}<0.05,{ }^{*} \mathrm{P}<0.01,{ }^{* * *} \mathrm{P}<0.001\right)$ compared with the wild-type when seedlings exposed to the same treatment time as determined by student's t test, ns indicates no significance. 

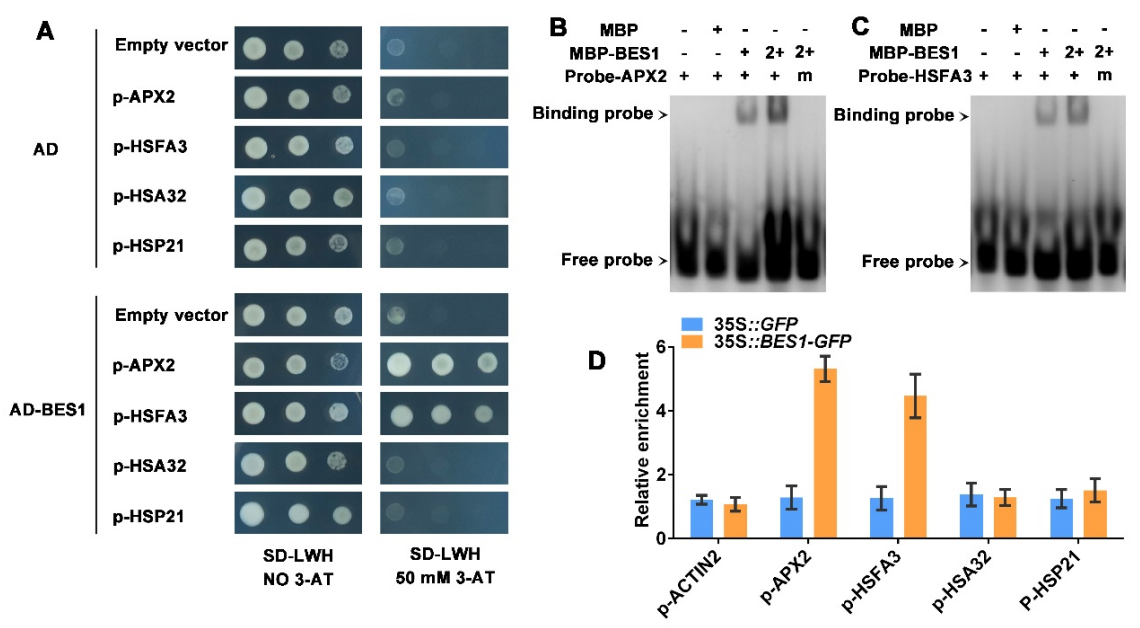

Figure 6. APX2 and HSFA3 are two direct binding targets of BES1.

(A). Activation of BES1 against promoters of HS-associated memory genes was tested in Y1H experiment. Growth of yeast transformants carrying the HIS3 reporter gene under the control of each promoter region was examined in the medium lacking leucine (L), Tryptophane (W) and histidine (H) with or without 3-AT.

(B). Electrophoretic mobility shift assay. MBP-BES1 protein binds specifically to the BES1 binding site within the APX2 promoter. 2+ represents twice the amount of protein, m represents the probe mutated BES1 binding site.

(C). Electrophoretic mobility shift assay. MBP-BES1 protein binds specifically to the BES1 binding site within the HSFA3 promoter. 2+ represents twice the amount of protein, m represents the probe mutated BES1 binding site.

(D). ChIP-qPCR assays indicate enrichment of APX2 and HSFA3 promoter in the immunoprecipitation products of BES1 protein. The ChIP assays were performed with chromatin prepared from $35 S:: G F P$ and 35S::BES1-GFP plants harvest at $12 \mathrm{~h}$ after heat priming, using an anti-GFP antibody. The precipitated DNA was analyzed by qPCR using the primer pairs of HS-associated memory genes. P-ACTIN2 was used as the negative control. Values are means \pm SD of three independent biological replicates. 